\title{
O impacto da pandemia do COVID-19 no atendimento odontológico infantojuvenil no Sistema Único de Saúde de João Pessoa - PB
}

The impact of the COVID-19 pandemic on children's dental care in the Unique Health System of João Pessoa - PB

El impacto de la pandemia COVID-19 en la atención dental infantil en el Único Sistema de Salud de João Pessoa - PB

Luciana Marina Coutinho de Andrade Ventura Ribeiro ORCID: https://orcid.org/0000-0003-3018-3744 Centro Universitário Maurício de Nassau, Brasil E-mail: lucianamaria@gmail.com Marta Martins Ferreira ORCID: https://orcid.org/0000-0002-3818-6256 Centro Universitário Maurício de Nassau, Brasil E-mail: martamartins@gmail.com

Jabes Gennedyr da Cruz Lima ORCID: https://orcid.org/0000-0001-7420-7686 Universidade Federal do Rio Grande do Norte, Brasil E-mail: jabes.gennedyr@hotmail.com

Danielle Machado Farias

ORCID: https://orcid.org/0000-0003-1829-0010 Universidade Federal do Rio Grande do Norte, Brasil E-mail: danifarias54@gmail.com

André Azevedo dos Santos

ORCID: https://orcid.org/0000-0002-8456-8394 Universidade Federal do Rio Grande do Norte, Brasil E-mail: andreazevedojs@gmail.com

Cristianne Kalinne Santos Medeiros

ORCID: https://orcid.org/0000-0003-2439-2523 Universidade Federal do Rio Grande do Norte, Brasil

E-mail: cristiannekalinne@gmail.com

Dennys Ramon de Melo Fernandes Almeida ORCID: https://orcid.org/0000-0002-4686-4379 Universidade Federal do Rio Grande do Norte, Brasil E-mail: dennysfernandes@ymail.com

Gabriel Coutinho Gonçalves

ORCID: https://orcid.org/0000-0002-5960-2976 Universidade Estadual do Ceará, Brasil E-mail: gabrielcoutinhoo@hotmail.com

Herrison Félix Valeriano da Silva ORCID: https://orcid.org/0000-0003-0601-6655 Faculdade Nova Esperança, Brasil E-mail: herrison.felix.vds@gmail.com

Suany Lara Soares Araújo ORCID: https://orcid.org/0000-0002-8290-8527 Centro Universitário Maurício de Nassau, Brasil E-mail: suanyaraujo2507@gmail.com

Juliana Campos Pinheiro ORCID: https://orcid.org/0000-0001-5687-7635 Universidade Federal do Rio Grande do Norte, Brasil E-mail: julianapinheiroodonto92@gmail.com

Rafaella Bastos Leite ORCID: https://orcid.org/0000-0002-3304-120X Centro Universitário Maurício de Nassau, Brasil E-mail: rrafaella_bastos@hotmail.com

Renata Dantas Barreto de Oliveira ORCID: https://orcid.org/0000-0001-6973-2283 Centro Universitário Maurício de Nassau, Brasil E-mail: renatadantas@gmail.com 


\begin{abstract}
Resumo
O presente estudo objetivou analisar o impacto da pandemia de coronavírus Sars-CoV-2 nos atendimentos odontológicos à população infantojuvenil na cidade de João Pessoa-PB. Trata-se de um estudo temporal realizado de setembro de 2019 à 2020. Foram utilizadas informações secundárias públicas aos serviços de atenção básica e secundária, caracterizadas pelos indicadores de saúde bucal os quais foram obtidos pelo SIA/SUS, banco de dados do Ministério da Saúde (DATASUS). Nesse estudo foram considerados os atendimentos odontológicos feitos no SUS, no semestre que antecedeu a pandemia de COVID-19. Nesse período, 1.413 procedimentos foram realizados em crianças e adolescentes na grande João Pessoa. Em média são realizados 235,5 \pm 85,3 procedimentos mensais. No primeiro semestre da pandemia do novo coronavírus, o número de procedimentos realizados apresentou uma queda de $46,42 \%$. Foram realizados 757 procedimentos odontológicos e no mês de maio, nenhum procedimento no público infantojuvenil foi referenciado. Dessa forma, nós podemos concluir que houve uma redução significativa no número de atendimentos odontológicos, e esse fenômeno pode gerar uma demanda reprimida significativa nesse público. Com isso nós sugerimos estudos para desenvolver políticas de prevenção e promoção de saúde bucal quando os atendimentos coletivos forem normalizados.
\end{abstract}

Palavras-chave: Infecções por Coronavirus; Odontopediatria; Odontologia em saúde pública.

\begin{abstract}
This study aimed to analyze the impact of the Sars-CoV-2 coronavirus pandemic on dental care for children and adolescents in the city of João Pessoa-PB. This is a temporal study carried out from September 2019 to 2020. Secondary public information was used for primary and secondary care services, characterized by oral health indicators which were obtained by SIA / SUS, a database of the Ministry of Health (DATASUS). In this study, dental care provided at SUS was considered in the semester that preceded the COVID-19 pandemic. During this period, 1,413 procedures were performed on children and adolescents in the greater João Pessoa. On average, $235.5 \pm 85.3$ monthly procedures are performed. In the first half of the new coronavirus pandemic, the number of procedures performed fell by $46.42 \% .757$ dental procedures were performed and in May, no procedures were performed in children and adolescents. Thus, we can conclude that there has been a significant reduction in the number of dental services, and this phenomenon can generate a significant pent-up demand in this audience. With that, we suggest studies to develop oral health promotion and prevention policies when collective care is normalized.
\end{abstract}

Keywords: Coronavirus infections; Pediatric dentistry; Public health dentistry.

\title{
Resumen
}

Este estudio tuvo como objetivo analizar el impacto de la pandemia del coronavirus Sars-CoV-2 en la atención odontológica de niños y adolescentes de la ciudad de João Pessoa-PB. Se trata de un estudio temporal realizado de septiembre de 2019 a 2020. Se utilizó información pública secundaria para los servicios de atención primaria y secundaria, caracterizada por indicadores de salud bucal que fueron obtenidos por SIA / SUS, base de datos del Ministerio de Salud (DATASUS). En este estudio, se consideró la atención odontológica brindada en el SUS en el semestre anterior a la pandemia de COVID-19. Durante este período, se realizaron 1.413 procedimientos en niños y adolescentes en el gran João Pessoa. En promedio, se realizan 235,5 \pm 85,3 procedimientos mensuales. En la primera mitad de la nueva pandemia de coronavirus, el número de procedimientos realizados cayó un 46,42\%. Se realizaron 757 procedimientos dentales y en mayo no se realizaron procedimientos en niños y adolescentes. Así, podemos concluir que se ha producido una reducción significativa en el número de servicios dentales, y este fenómeno puede generar una importante demanda reprimida en este público. Con eso, sugerimos estudios para desarrollar políticas de prevención y promoción de la salud bucal cuando se normalice el cuidado colectivo.

Palabras clave: Infecciones por Coronavirus; Odontología pediátrica; Odontología en salud pública.

\section{Introdução}

Com o novo coronavírus Sars-CoV-2 a Odontologia representa uma área importante de combate a esse novo tipo de doença infecciosa. Vale ressaltar que o profissional da odontologia Cirurgião Dentista é a pessoa que tem maior contato com a boca, nariz e complexo orofaríngeo do paciente. Além disso, as próprias secreções salivares estão em contato direto com os profissionais durante o serviço, por exemplo, na forma de aerossóis dispersos no ar do ambiente de trabalho (Pejic-Bach, 2020).

No Brasil, o primeiro caso foi confirmado em 25 de fevereiro, tornando-se o primeiro país da América Latina a relatar um caso de coronavírus. No dia 20 de março, o país reconheceu que a doença havia se espalhado na comunidade e, desde então, o Ministério da Saúde (MS) recomenda medidas de isolamento social para toda a população (Choi, 2020; Pejic-Bach, 2020). 
O surgimento da pandemia do COVID-19 provocou uma mudança de hábitos na vida das pessoas em todo o mundo, afetando e trazendo muitos desafios nos aspectos pessoais, econômicos, públicos, privados e empresariais. Tal mudança fez com que entidades públicas e privadas tivessem que mudar toda a logística da oferta de seus produtos e serviços. Quarentenas foram adotadas, o que causou a diminuição do contato físico entre as pessoas (Cao, 2020; Pejic-Bach, 2020).

Durante a pandemia do COVID-19, as mudanças na situação epidemiológica do país não afetam apenas os profissionais que prestam serviços médicos diretos relacionados à doença, mas também outros profissionais e pessoas que utilizam os serviços (Cook, 2020; Pejic-Bach, 2020).

A Agência Nacional de Vigilância Sanitária (ANVISA), vem publicando notas técnicas, que são atualizadas de acordo com o decorrer da pandemia, onde foram estabelecidas regras para assistência odontológica em todo país, no documento foi diferenciado os cuidados em diferentes cenários: consultório/ambulatório, o ambiente hospitalar e a unidade de terapia intensiva. $\mathrm{O}$ documento também fez uma restrição aos procedimentos odontológicos, dando ênfase ao atendimento de urgência e emergência, anamnese criteriosa, salas de espera contendo equipamentos de proteção individual, recomendou realizar previamente ao atendimento bochecho com peróxido de hidrogênio 1 a 1,5\% e dar preferência ao uso de dispositivos manuais durante o atendimento para evitar gerar aerossóis (Banihani, 2018; Pejic-Bach, 2020).

Para atendimento odontológico, conforme Nota Técnica n ${ }^{\circ}$ 9/2020, a recomendação é suspender o atendimento eletivo e manter os atendimentos emergenciais que devem ser realizadas de forma individual, para evitar a disseminação do vírus. Tais medidas de proteção aos profissionais e usuários dos serviços de saúde, além de contribuir para o aumento da demanda reprimida, também impactarão os indicadores desses serviços (como indicadores de acesso e resolutividade) (Sigua-Rodrigues, 2020; Pejic-Bach, 2020).

O Conselho Federal de Odontologia (CFO) ressaltou a divisão dos atendimentos de urgência e emergência que deverão ser atendidos durante período pandêmico. São caracterizados como urgência: Pulpite irreversível, Pericoronanite, osteíte pós-operatória cirúrgica ou troca de curativos de cavidade seca, abscesso ou infecção bacteriana localizada, resultando em dor e inchaço localizados, fratura de dente resultando em dor ou causando trauma nos tecidos moles, trauma dentário com avulsão / luxação, confecção de restauração temporária caso a restauração for perdida, quebrada ou esteja causando irritação gengival, cárie extensa ou restaurações defeituosas que causam dor, remoção de suturas, ajustes da dentadura em pacientes em radiação / tratamento oncológico, mucosites, ajustes ou reparos da dentadura quando a função é impedida, substituição do preenchimento temporário nas aberturas de acesso endodôntico em pacientes com dor e corte ou ajustes de um fio ou aparelhos ortodônticos que perfuram ou ulceram a mucosa bucal. São caracterizados como atendimentos de emergência: Sangramento descontrolado, celulite ou infecção difusa de tecidos moles com potencial comprometimento das vias aéreas do paciente, trauma envolvendo os ossos da face com potencial comprometimento das vias aéreas do paciente (Al-Halabi, 2018; PejicBach, 2020).

Os atendimentos eletivos foram suspensos, e mesmo para os atendimentos de urgência e emergência, tornou-se necessário, uma adequação dos cirurgiões dentistas, frente a biossegurança. Novas medidas de biossegurança que antes não eram preconizadas, tornou-se rotina obrigatória nos atendimentos clínicos, além dos cirurgiões dentistas outras profissões outros profissionais que antes não utilizavam Equipamentos de Proteção Individual (EPI), passaram a utilizar, aumentando a demanda, fazendo com que os valores se elevassem, o que acabou dificultando, ainda mais o retorno dos atendimentos eletivos, devido aos altos valores dos EPIs (Sigua-Rodrigues, 2020; Texeira, 2020).

No atendimento odontológico diversas recomendações foram feitas, algumas delas são: merecendo destaque para o uso de mascaras N-95 descartáveis para o atendimento, o uso de aventais descartáveis impermeáveis, o uso de face Shields, uso de touca descartável impermeável de gramatura superior a $30 \mathrm{~g} / \mathrm{m}^{2}$, o uso de óculos de proteção com vedamento lateral, bem como a diminuição sempre que possível do uso da caneta de alta rotação, pela liberação de aerossóis, que podem 
contaminar até um raio de 1,5 metro e permanecer pairando no ar por um período de até 3 horas, aumentando o risco de infecção cruzada entre que possam contaminar os profissionais e seus respectivos pacientes (Sigua-Rodrigues, 2020).

A adaptação às necessidades de novos procedimentos odontológicos e o alto custo dos equipamentos de proteção individual podem influenciar na redução do número de atendimentos odontológicos (Ge, 2020; Meng, 2020). Portanto, é possível sugerir que os cuidados com a saúde bucal enfrentarão grandes desafios durante e após a pandemia de coronavírus, embora o foco esteja no ambiente hospitalar durante este período, o fortalecimento da atenção primária como um todo é essencial para lidar com esses impactos (Ge, 2020; Meng, 2020).

O COVID-19 é um vírus que se transmite de forma muito rápida entre as pessoas, trata-se de É um vírus novo que vem apresentando consequências imprevisíveis, ainda não existe vacina ou medicamento para o controle da patologia. Em reflexo a pandemia, os governantes introduziram medidas restritivas, afetando a vida diária da população, incluindo a restrição no atendimento odontológico, consequentemente causando interrupções consideráveis nos serviços de saúde bucal (Banihani, 2020).

A COVID-19 trouxe um desafio para os atendimentos odontológicos, tendo em vista que o índice de contágio entre os profissionais da saúde é elevado. O cirurgião dentista tem grande possibilidade de adquirir e disseminar a infecção, devido ao contato próximo com o paciente e a presença constante de aerossóis durante os atendimentos. Com o objetivo de controlar e evitar a disseminação da doença o profissional deve ter conhecimento suficiente para realizar o atendimento clínico de forma segura (Kenyon, 1996; Sigua-Rodrigues, 2020).

A Odontologia modificou o seu entendimento sobre o tratamento das doenças bucais, passando de um atendimento direcionado para realização de procedimentos curativos, para um atendimento voltado ao processo saúde-doença. Essa mudança mostrou a importância do atendimento precoce da população, visando minimizar sequelas causadas pelas principais doenças bucais. Diante dessa perspectiva o atendimento na primeira infância torna-se de extrema importância na prevenção e recuperação de agravos decorrentes pela cárie dentária (Berg, 2002; Essvein, 2019).

Sabe-se que é de extrema importância, o acompanhamento odontológico já nos primeiros anos de vida das crianças, para prevenir e tratar doenças futuras, a odontologia atual tem como base, fazer a prevenção para evitar tratamentos no futuro, sabendo-se disso torna-se importantíssimo, orientar os pais e responsáveis de crianças, a fazer um acompanhamento odontológico, das suas crianças, desde dos primeiros anos de vida, pois com isso podemos prevenir que essas crianças venham a desenvolver diversas alterações bucais no futuro (Bloch, 1985; Essvein, 2019).

A cárie dentaria é umas das doenças crônicas mais prevalentes, sendo considerada mundialmente um grave problema de saúde pública. Com prevalência de aproximadamente 53,4\% em crianças de cinco anos de idade, também podemos destacar que $70 \%$ das crianças com até 12 anos já apresentaram cárie em ao menos um dente. Durante a primeira infância as condições de saúde inadequadas têm grande impacto negativo na saúde geral desencadeando comprometimentos biopsicossociais (Essvein, 2019).

Com o surgimento da coronavírus, os odontopediatras enfrentam dificuldades nunca vividas. Cerca de $2 \%$ total dos casos são representados por crianças menor de 16 anos, em grande maioria assintomáticas, o que pode contribuir para transmissão da doença e gerar grande preocupação para os profissionais, devido à incerteza do seu potencial infeccioso. Diante disso, torna-se relevante lembrar que atender criança é desafiador, pois elas podem chorar, tossir, espirar, podendo gerar aerossóis durante o tratamento odontológico (Abu-Serdaneh, 2020; Banihani, 2020).

É esperado que essa doença permaneça por um longo prazo, com uma segunda onda de casos. Analisando o aumento na proporção de crianças com cárie dentária em todo o mundo e as consequências negativas que isso gera em sua qualidade de vida, durante o período de pandemia, alternativas ao tratamento dentário convencional, podem ser reconsideradas em dentes decíduos cariados, podendo ser incluída a retirada não seletiva da cárie dentária, como também a terapia da polpa usando peça 
de mão de alta rotação e seringa 3 em 1, visando diminuir a produção de aerossóis e manter um ambiente mais seguro e limpo para a equipe e os pacientes (Banihani, 2020). Nesse contexto, o presente estudo objetivou analisar o impacto da pandemia de coronavírus Sars-CoV-2 nos atendimentos odontológicos à população infantojuvenil na cidade de João Pessoa-PB.

\section{Metodologia}

Esta pesquisa é caracterizada como um estudo descritivo, utilizando dados secundários, descritivo, temporal e com abordagem quantitativa das cinco faixas etárias da infância e juventude: (menor que 1 ano, 1 a 4 anos, 5 a 9 anos, 10 a 14 anos e 15 a 19 anos). O mês de março de 2019 não foi considerado por ser o mês do início da pandemia, onde houve a transição na forma de atendimento odontológico na rede pública de saúde. Dessa forma, seis meses de atendimento foram incluídos no período pré-pandemia (setembro/2019, outubro/2019, novembro/2019, dezembro/2019, janeiro/2020 e fevereiro/2020) e seis meses do período de pandemia (abril/2020, maio/2020, junho/2020, julho/2020, agosto/2020, setembro/2020)

Foram utilizadas informações públicas aos serviços de atenção básica à saúde bucal, caracterizadas pelos indicadores, a fim de identificar o tipo de atenção em saúde bucal adotada no Brasil no período prévio e durante a pandemia do COVID-19. Os dados referentes aos indicadores de saúde bucal foram obtidos pelo SIA/SUS, banco de dados do Ministério da Saúde (DATASUS), no site http://www.datasus.gov.br, e no Instituto Brasileiro de Geografia e Estatísticas (IBGE).

A coleta de dados foi realizada no site do DATASUS (http://datasus.saude.gov.br/) utilizando os seguintes filtros: acesso a informação, informação em saúde (TABNET), grupo produção ambulatorial (SIA/SUS), local de atendimento (João Pessoa - PB) e os procedimentos realizados, quem foram divididos em invasivos: (tratamento endodôntico de dente decíduo; tratamento endodôntico de dente permanente birradicular; tratamento endodôntico de dente permanente com três ou mais raízes; tratamento endodôntico de dente permanente anterior; pulpotomia dentária; retratamento endodôntico em dente permanente birradicular; retratamento endodôntico em dente permanente com 3 ou mais raízes; retratamento endodôntico em dente permanente uni-radicular; exodontia de dente decíduo; exodontia de dente permanente); conservadores: (restauração de dente decíduo; restauração de dente permanente anterior com resina composta; restauração de dente permanente posterior; tratamento restaurador atraumático (tra/art); restauração de dente decíduo posterior com amálgama; restauração de dente decíduo posterior com ionômero de vidro; restauração de dente decíduo anterior com resina composta; restauração de dente permanente posterior com resina composta; remoção/restauração com amálgama de dente permanente posterior; raspagem alisamento e polimento supragengivais (por sextante); raspagem alisamento subgengivais (por sextante); restauração de dente decíduo posterior com resina composta).

Foi escolhida a "quantidade apresentada", pois esse modo de registro contém todos os procedimentos informados ao sistema. Além dos dados do SIA/SUS, foram necessários, os dados sobre a população presente das regiões no ano de 2019 e 2020, obtidos pelo IBGE. Os dados referentes a produção ambulatorial foram tabulados por meio do software TabWin, versão 3.52, disponibilizado pelo DATASUS. Após a tabulação, os dados foram exportados para o programa Microsoft Office Excel®, e analisados pelos autores.

\section{Resultados}

O município de João Pessoa - Paraíba, possui uma população estimada de 800.323 habitantes. Nesta pesquisa foram considerados os atendimentos odontológicos feitos no SUS, na atenção primária e secundária, no semestre que antecedeu a pandemia de COVID-19, na população infantojuvenil. Nesse período, 1.413 procedimentos foram realizados em crianças e adolescentes na grande João Pessoa. Em média são realizados 235,5 \pm 85,3 procedimentos mensais (Tabela 1). 
Tabela 1 - Números de procedimentos odontológicos realizados no município de João Pessoa - PB no semestre que antecedeu a pandemia de COVID-19.

\begin{tabular}{|l|l|l|l|l|l|l|}
\hline Faixa etária & $\mathbf{0 9 / 1 9}$ & $\mathbf{1 0 / 1 9}$ & $\mathbf{1 1 / 0 9}$ & $\mathbf{1 2 / 1 9}$ & $\mathbf{0 1 / 2 0 2 0}$ & $\mathbf{0 2 / 2 0 2 0}$ \\
\hline Menor 1 ano & - & - & - & 1 & 2 & 9 \\
\hline 1 a 4 anos & 19 & 22 & 21 & 23 & 16 & 22 \\
\hline 5 a 9 anos & 48 & 81 & 33 & 34 & 34 & 42 \\
\hline 10 a 14 anos & 143 & 111 & 41 & 68 & 51 & 108 \\
\hline 15 a 19 anos & 139 & 93 & 70 & 32 & 56 & 94 \\
\hline TOTAL & $\mathbf{3 4 9}$ & $\mathbf{3 0 7}$ & $\mathbf{1 6 5}$ & $\mathbf{1 5 8}$ & $\mathbf{1 5 9}$ & $\mathbf{2 7 5}$ \\
\hline
\end{tabular}

Fonte: Autores.

No primeiro semestre da pandemia do novo coronavírus, o número de procedimentos realizados apresentou uma queda de 46,42\%. Foram realizados 757 procedimentos odontológicos e no mês de maio, nenhum procedimento no público infantojuvenil foi referenciado (Tabela 2).

Tabela 2 - Números de procedimentos odontológicos realizados no município de João Pessoa - PB no primeiro semestre da pandemia de COVID-19.

\begin{tabular}{|l|l|l|l|l|l|}
\hline Faixa etária & 2020/Abr & 2020/Jun & 2020/Jul & 2020/Ago & 2020/Set \\
\hline Menor 1 ano & - & - & 1 & - & - \\
\hline 1 a 4 anos & 24 & - & - & 19 & 11 \\
\hline 5 a 9 anos & 32 & - & 1 & 48 & 120 \\
\hline 10 a 14 anos & 33 & - & 1 & 43 & 86 \\
\hline 15 a 19 anos & 73 & 8 & 25 & 78 & 154 \\
\hline TOTAL & $\mathbf{1 6 2}$ & $\mathbf{8}$ & $\mathbf{2 8}$ & $\mathbf{1 8 8}$ & $\mathbf{3 7 1}$ \\
\hline
\end{tabular}

Fonte: Autores.

Durante o período pré-pandemia, o número de procedimento invasivos relatados no DATASUS contabilizou o número de 18, sendo que no mês de janeiro, nenhum procedimento foi relatado (Tabela 3).

Tabela 3 - Números de procedimentos odontológicos “invasivos” realizados no município de João Pessoa - PB no semestre que antecedeu a pandemia de COVID-19.

\begin{tabular}{|l|l|l|l|l|l|l|l|} 
Faixa etária & \multicolumn{2}{l}{ 2019/Set } & 2019/Out & 2019/Nov & 2019/Dez & 2020/Jan & 2020/Fev \\
\hline 1 a 4 anos & - & - & - & - & - & - \\
\hline 5 a 9 anos & 3 & 3 & - & 3 & - & 2 \\
\hline 10 a 14 anos & - & 4 & - & 1 & - & - \\
\hline 15 a 19 anos & - & 1 & 1 & - & - & - \\
\hline TOTAL & $\mathbf{3}$ & $\mathbf{8}$ & $\mathbf{1}$ & $\mathbf{4}$ & - & $\mathbf{2}$ \\
\hline
\end{tabular}

Fonte: Autores. 
No primeiro semestre da pandemia, apenas nos meses de agosto e setembro foram realizados procedimentos, sendo que em cada um desses meses, apenas um procedimento foi relatado (Tabela 4).

Tabela 4 - Números de procedimentos odontológicos “invasivos” realizados no município de João Pessoa - PB no primeiro semestre que da pandemia de COVID-19.

\begin{tabular}{|l|l|l|}
\hline Faixa etária & 2020/Ago & - \\
\hline 1 a 4 anos & 1 & - \\
\hline 5 a 9 anos & - & - \\
\hline 10 a 14 anos & - & - \\
\hline 15 a 19 anos & 1 & 1 \\
\hline TOTAL & 1 & 1 \\
\hline
\end{tabular}

Fonte: Autores.

Quanto aos procedimentos odontológicos "conservadores", no semestre que antecedeu a pandemia do novo coronavírus, 434 procedimentos foram realizados. Uma média de $72 \pm 20$ procedimentos realizados mensalmente no público infantojuvenil (Tabela 5).

Tabela 5 - Números de procedimentos odontológicos “conservadores” realizados no município de João Pessoa - PB no semestre que antecedeu a pandemia de COVID-19.

\begin{tabular}{|c|c|c|c|c|c|c|}
\hline Faixa etária & 2019/Set & 2019/Out & 2019/Nov & 2019/Dez & 2020/Jan & 2020/Fev \\
\hline 1 a 4 anos & 1 & 5 & 6 & 20 & 4 & 4 \\
\hline 5 a 9 anos & 10 & 26 & 7 & 17 & 12 & 16 \\
\hline 10 a 14 anos & 43 & 47 & 16 & 41 & 17 & 42 \\
\hline 15 a 19 anos & 19 & 16 & 24 & 13 & 11 & 17 \\
\hline TOTAL & 73 & 94 & 53 & 91 & 44 & 79 \\
\hline
\end{tabular}

Fonte: Autores.

No primeiro semestre da pandemia do novo coronavírus, foram relatados procedimentos odontológicos conservadores em apenas três meses: julho, agosto e setembro, totalizando o número de 44 procedimentos. Destes, o mês de agosto foi o que relatou maior número de procedimentos, correspondendo a $63 \%$ dos atendimentos (Tabela 6).

Tabela 6 - Números de procedimentos odontológicos “conservadores” realizados no município de João Pessoa - PB no primeiro semestre da pandemia de COVID-19.

\begin{tabular}{|l|l|l|l|}
\hline Faixa etária & 2020/Jul & 2020/Ago & \multicolumn{2}{l|}{ 2020t } \\
\hline 1 a 4 anos & - & 10 & - \\
\hline 5 a 9 anos & - & 3 & 2 \\
\hline 10 a 14 anos & 1 & 6 & - \\
\hline 15 a 19 anos & - & 9 & 13 \\
\hline TOTAL & $\mathbf{1}$ & $\mathbf{2 8}$ & $\mathbf{1 5}$ \\
\hline
\end{tabular}

Fonte: Autores. 


\section{Discussão}

Os indicadores de atendimento são de extrema importância para que se torne possível avaliar as práticas odontológicas preventivas e curativas que estão sendo realizadas em um determinado local, em especial nas unidades básicas de saúde. Pode-se constatar, no presente estudo, que houve uma queda considerável na realização dos atendimentos odontológicos infantojuvenil, na atenção primária e secundária do SUS, no município de João pessoa, durante os seis primeiros meses do início da pandemia em relação aos seis meses que antecederam a pandemia. Esse resultado torna-se preocupante, pois essa constante e a nítida redução dos atendimentos odontológicos, contribui para que haja uma significativa demanda reprimida em diferentes níveis de complexidade (Al-Halabi, 2020; Banihani, 2020).

Em um estudo feito por Mendonça (2017), é relatado que as lesões iniciais da cárie se apresentam de forma assintomática, momento em que poderão ser tradadas por meio de tratamentos conservadores e indolores. As manchas brancas na superfície dental, são as primeiras manifestações clínicas, se essa não for controlada, ocorrerá cavitação da superfície. No paciente infantil, na maioria dos casos, essas lesões não são identificadas pelos pais, que só notarão a presença da doença quando a mesma evoluir para casos de desconforto, dor ou problema estético.

A cárie dentaria é uma doença multifatorial, sua dinâmica depende basicamente da integração e presença de quatro fatores predisponentes, sendo eles: dieta, microbiota, hospedeiro e tempo, estando presente esses fatores, ocorre uma alteração no $\mathrm{pH}$ do meio bucal, que leva a um desequilíbrio na cavidade, ocorrendo a perda de minerais do esmalte para o meio bucal. Quando esta situação persiste por algum tempo, haverá o desenvolvimento das lesões de cárie (Harrel, 2004; Mendonça, 2017). Diante disto, podemos sugerir que o cancelamento, provocados pela pandemia, nos atendimentos odontológicos eletivos poderão contribuir significativamente para um grande aumento nas estatísticas dos índices de cárie dental na população infantojuvenil.

Outro ponto relevante a ser analisado é que nesse estudo pode-se constatar que, no município de João Pessoa, durante o período de pré-pandemia, já havia uma baixa demanda para procedimentos "invasivos", onde com isso podemos sugerir que o atendimento na atenção primária está sendo realizado de forma rápida e eficiente, suprindo as necessidades básicas para que os pacientes não necessitem de procedimentos que são considerados mais complexos, onde haja risco de perca do elemento dentário.

Como foi possível observar, durante a pandemia, o número de atendimentos odontológicos "invasivos" sofreu uma queda drástica. Em nossa Tabela 4, analisamos os atendimentos durante os meses de agosto e setembro, período equivalente ao início do primeiro semestre da pandemia e constatamos que na faixa etária de 1 a 4 anos, não houve nenhum atendimento odontológico com procedimentos invasivos. Na faixa etária de 5 a 9 anos, foi constatado apenas 1 procedimento no mês de agosto e 1 procedimento no mês de setembro. Já nas faixas etárias de 10 a 14 e 15 a 19 também não foram realizados nenhum tipo de procedimentos invasivos neste período.

Outra questão que também deve ser levada em consideração e que confirma a eficácia no atendimento odontológico primário, é que no município de João Pessoa a água de abastecimento público não é fluoretada (Mendonça, 2017). A fluoretação das águas de abastecimento público é vista como o melhor método de exposição coletiva ao flúor, pois tem um baixo custo, uma alta frequência de consumo e uma alta efetividade. Ainda é considerado, na saúde pública, o método mais significativo do uso do flúor. Há evidências que em comunidades que fazem uso contínuo de água fluoretada, existe uma expectativa de redução entre 50 a $65 \%$ da prevalência de cárie dentária, assim evidenciando a sua relevância pública e o seu comprovado efeito anticárie (Garner, 1996; Banihani, 2020).

Tratando-se de procedimentos conservadores, na tabela 5, pode ser observado durante os meses de setembro de 2019 à fevereiro de 2020, semestre que antecedeu o início da pandemia, houve uma totalidade de 434 procedimentos realizados, já no 
semestre seguinte, durante a pandemia, este número de procedimentos caiu para 44 procedimentos, como pode ser observado na tabela 6. Nota-se que essa diminuição de atendimentos foi maior a partir do mês de abril, tendo em vista que foi o período onde o número de casos de coronavírus subiu, e houve uma certa restrição nos atendimentos odontológicos, onde os atendimentos só aconteciam em casos de urgência e emergência, este cenário é extremamente preocupante, tendo em vista que, o acompanhamento odontológico, principalmente nos primeiros anos de vida da criança é de extrema importância e, sabe-se que a falta desse acompanhamento pode acarretar grandes problemas na saúde bucal das crianças.

Demarco (2020), em seus trabalhos defende que as lesões por cárie tendem a progredir de forma mais rápida em crianças, pois o esmalte, que é um dos tecidos que compõe os dentes, é menos mineralizado em crianças que em adultos.

O CPOD é um índice recomendado pela Organização Mundial da Saúde (OMS) para mensurar e comparar a existência de cárie dentária na população, seu valor traz informações sobre a média de dentes cariados, perdidos e obturados em um grupo de indivíduos. No ano de 2003 foi realizado um levantamento epidemiológico na região nordeste e foi constatado a média de CPOD de 3,19 aos 12 anos de idade e, em uma pesquisa realizada no ano de 2006 em escolas públicas e privadas na cidade de João Pessoa, constatou-se o nível de CPOD, aos 12 anos de idade, para os alunos de escola públicas de 3,37 e alunos das escolas privadas de 1,35 (Moreira, 2007).

É previsível que devido a pandemia e a redução dos atendimentos odontológicos infantis, haja um aumento significante dos problemas na saúde bucal de todo o público infantil, refletindo em um aumento do número de elementos cariados, perdidos e obturados nas crianças, impactando negativamente na saúde das crianças de João Pessoa, tendo em vista que, a cárie seguida das doenças periodontais é uma das doenças mais prevalentes na população, sendo um dos maiores motivos de perdas dentárias, condição esta, que traz enormes malefícios na qualidade de vida dos indivíduos.

\section{Conclusão}

Com base nessa pesquisa, que utilizou dados secundários e considerando as limitações inerentes a esse tipo de abordagem metodológica, concluímos que houve uma queda abrupta no atendimento odontológico ao público infantojuvenil na atenção básica e secundária no município de João Pessoa - PB, durante a pandemia do coronavírus. Essa demanda suprimida acomete principalmente as classes sociais menos favorecidas, que já possuem um CEO-D e CPO-D mais elevado.

Sugerimos novos estudos que visem a criação de campanhas educativas que se adequem ao nova cenário que estamos enfrentando, para incentivar os pais e responsáveis legais da população infantojuvenil, a realizar a higiene oral adequada, visando evitar o surgimento de doenças relacionadas ao meio bucal, assim podendo evitar a necessidade imediata de um acompanhamento de um cirurgião dentista e também de planos de ação que visem minimizar as sequelas deixadas por esse período.

\section{Referências}

Abu-Serdaneh, S. (2020). Coroas com técnica de Hall e atividade do músculo masseter infantil: estudo piloto de eletromiografia de superfície. Int J Paediatr Dent. 30 (3): 303-313.

Al-Halabi, M. (2018). Alternativas restauradoras de molares primários biológicos híbridos amigáveis à criança para anestesia geral. Atualização Dent. 2018 (45):728-741.

Al-Halabi, M. (2020). Assessment of paediatric dental guidelines and caries management alternatives in the post COVID-19 period. A critical review and clinical recommendations. Eur Arch Paediatr Dent. 10(10):1.

Banihani A. (2018). Outcomes of the conventional and biological treatment approaches for the management of caries in the primary dentition. Int J Paediatr Dent. 28(1):12-22.

Banihani, A. (2020). Could COVID-19 change the way we manage caries in primary teeth? Current implications on Paediatric Dentistry. 30(5): 523-525.

Berg, J. H. (2002). Glass ionomer cements. Pediatr Dent. 24(1):430-438. 
Research, Society and Development, v. 10, n. 5, e17110515089, 2021

(CC BY 4.0) | ISSN 2525-3409 | DOI: http://dx.doi.org/10.33448/rsd-v10i5.15089

Bloch, A. B. (1985). Measles outbreak in a pediatric practice: airborne transmission in an office setting. Pediatrics. 75(1):676-683.

Cao, Q. (2020). SARS-CoV-2 infection in children: transmission dynamics and clinical characteristics. J Formos Med Assoc. 119(1):670-673.

Choi, S. H. (2020). Epidemiology and clinical features of coronavirus disease 2019 in children. Clin Exp Pediatr. 63(4):125-132.

Cook, T. M. (2020). Personal protective equipment during the coronavirus disease (COVID) 2019 pandemic a narrative review. Anaesthesia. 75(7):920-927.

Essvein, G. (2019). Atendimento odontológico na primeira infância no Brasil: da política pública à evidência. Rev. Saúde Pública. 53(15):10.

Garner, J. S. (1996). Guideline for isolation precautions in hospitals. The Hospital Infection Control Practices Advisory Committee. Infect Control Hosp Epidemiol. 17(1):53-80.

Ge, Y. L. (2020). Possible aerosol transmission of COVID-19 and special precautions in dentistry. Zhejiang Univ-Sci B (Biomed \& Biotechnol). 21(5):361368.

Harrel, S. K. (2004). Aerosols and splatter in dentistry. A brief review of the literature and infection control implications. JADA. 135(1):429-437.

Kenyon, T. A. (1996). Transmission of multidrug-resistant Mycobacterium tuberculosis during a long airplane flight. N Engl J Med. 334(15):933-938.

Mendonça, J. G. A. (2017). Impacto do tratamento odontológico na qualidade de vida de um grupo de escolares brasileiros. 2017. 63 f., il. Dissertação (Mestrado em Ciências da Saúde). Universidade de Brasília, Brasília, 2017. https://repositorio.unb.br/handle/10482/31811.

Meng, L. (2020). Coronavirus Disease 2019 (COVID-19): Emerging and Future Challenges for Dental and Oral Medicine. J Dent Res. 99(5):481-487.

Moreira, P. V. L. (2007). Prevalência de cárie em adolescentes de escolas públicas e privadas na cidade de João Pessoa, Paraíba, Brasil. Ciênc. saúde coletiva. 12(5): $1229-1236$

O'Hoorey, D. (2020). The Aerosol generating procedure: how a phrase lost it's way within the maze of COVID-19 and dentistry. Dent Update. 47(1):471-475.

Sigua-Rodrigues, E. A. (2020). COVID-19 y la Odontología: una Revisión de las Recomendaciones y Perspectivas para Latinoamérica. Int. J. Odontostomat. Тетисо, 14(3): 299-309. 